\title{
Pacemaker lead-induced tricuspid regurgitation: consider leaflet remodeling
}

\author{
Federico Fortuni ${ }^{1} \cdot$ Frank van der Kley $^{1} \cdot$ Victoria Delgado $^{1} \cdot$ Nina Ajmone Marsan ${ }^{1}$
}

Received: 2 December 2020 / Accepted: 4 December 2020 / Published online: 27 January 2021

(c) The Author(s) 2021

Cardiac implantable electronic device (CIED) can interfere with tricuspid valve (TV) function, induce significant tricuspid regurgitation (TR), and worsen patient prognosis [1]. We present a case of pacemaker (PM) lead induced severe TR (Fig. 1, Panel A-B) that led to progressive right atrial dilation and heart failure (HF) symptoms 2 years after PM implantation. Transthoracic 2- and 3-dimensional echocardiograms demonstrated a mechanical compression of the TV septal leaflet by the PM lead (arrow on Panel B and D of Fig.1 and Supplemental Video). The patient underwent lead extraction and a leadless PM was positioned at the apex of the right ventricle (RV). Intracardiac echocardiography was performed during the procedure and showed persistence of severe TR after lead extraction (Fig. 1, Panel E) due to retraction (without rupture) of the septal leaflet (asterisk on Panel E of Fig. 1). Transthoracic echocardiography confirmed these findings and showed no significant RV remodeling (Fig. 1, Panel C). Due to persistence of HF symptoms, the patient underwent surgical TV replacement and the intra-operative inspection confirmed an isolated significant thickening and fibrosis of the TV septal leaflet (without rupture) probably induced by the mechanical compression of the PM lead. This case underlines the importance of aiming for a CIED lead position that does not interfere with the TV apparatus to avoid development of TR. Furthermore, we suggest careful imaging and clinical evaluation of patients who develop TR after CIED implantation to optimize the timing for lead repositioning, since TR may not be reversible after lead extraction and surgical correction is needed if irreversible damages of the TV leaflets have occurred.

Supplementary information The online version of this article (https://doi.org/10.1007/s10554-020-02131-w) contains supplementary material, which is available to authorized users.

Nina Ajmone Marsan

N.Ajmone@lumc.nl

1 Department of Cardiology, Heart LungCenter, Leiden University Medical Center, Albinusdreef 2, 2300 RC Leiden, The Netherlands 


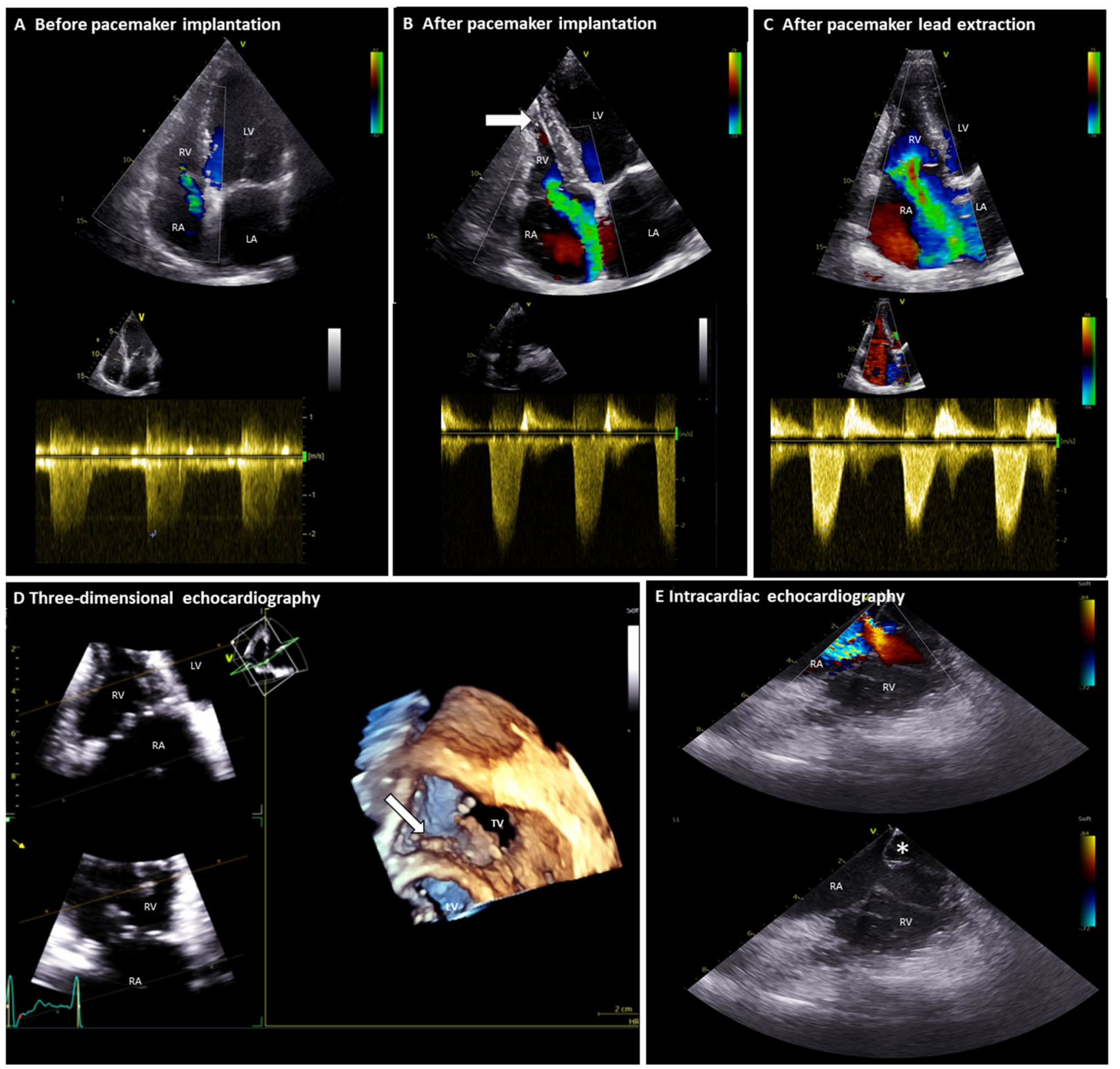

Fig. 1 Pacemaker lead-induced tricuspid regurgitation. $L A$ left atrium, $L V$ left ventricle, $R A$ right atrium, $R V$ right ventricle, $T V$ tricuspid valve 
Funding This work did not receive any specific funding.

\section{Compliance with ethical standards}

Conflict of interest The Department of Cardiology of the Leiden University Medical Center received research grants from Abbott Vascular, Bayer, Bioventrix, Medtronic, Biotronik, Boston Scientific, GE Healthcare, Edwards Lifesciences and Medtronic. Nina Ajmone Marsan received speaking fees from Abbott Vascular and GE healthcare. Victoria Delgado received speaker fees from Abbott Vascular, Medtronic, MSD, Edwards Lifesciences and GE Healthcare. The remaining authors have nothing to disclose.

Open Access This article is licensed under a Creative Commons Attribution 4.0 International License, which permits use, sharing, adaptation, distribution and reproduction in any medium or format, as long as you give appropriate credit to the original author(s) and the source, provide a link to the Creative Commons licence, and indicate if changes were made. The images or other third party material in this article are included in the article's Creative Commons licence, unless indicated otherwise in a credit line to the material. If material is not included in the article's Creative Commons licence and your intended use is not permitted by statutory regulation or exceeds the permitted use, you will need to obtain permission directly from the copyright holder. To view a copy of this licence, visit http://creativecommons.org/licenses/by/4.0/.

\section{Reference}

1. Addetia K, Harb SC, Hahn RT, Kapadia S, Lang RM (2019) Cardiac implantable electronic device lead-induced tricuspid regurgitation. JACC Cardiovasc Imaging 12:622-636

Publisher's Note Springer Nature remains neutral with regard to jurisdictional claims in published maps and institutional affiliations. 